\section{Análisis de variabilidad del ritmo cardiaco y su relación con la sensibilidad insulínica en pacientes obesos y con sobrepeso}

\author{
ALEXIS ESPINOZA-SALINAS ${ }^{1, \mathrm{a}}$, EDSON ZAFRA-SANTOS ${ }^{1}$, \\ GUSTAVO PAVEZ-VON MARTENS ${ }^{1, b}$, CRISTIAN COFRÉ-BOLADOS ${ }^{2, \mathrm{c}}$, \\ JORGE LEMUS-ZÚÑIGA ${ }^{1, d}$, PABLO SÁNCHEZ-AGUILERA ${ }^{1, \mathrm{~d}}$
}

\section{Heart rate variability and insulin resistance among obese males}

Background: Heart rate variability analysis provides quantitative information about vagal and sympathetic modulation of cardiac function. Aim: To analyze the relationship between heart rate variability and insulin resistance in obese patients. Material and Methods: Male participants were studied, divided in 10 obese subjects aged $27 \pm 2$ years with a body mass index (BMI) of $31.2 \pm 1.3$ $\mathrm{kg} / \mathrm{m}^{2}, 15$ overweight subjects aged $24 \pm 3$ years with a BMI of $26.7 \pm 1.5 \mathrm{~kg} / \mathrm{m}^{2}$ and 14 normal weight subjects aged $21 \pm 2$ years with a BMI of $22.5 \pm 1.3 \mathrm{~kg} / \mathrm{m}^{2}$. Resting heart rate variability was measured in a period of 5 minutes. A spectral analysis was done measuring the low frequency/high frequency ratio (LF/HF). A non-linear analysis was carried out measuring the standard deviation of the instantaneous variation of $R R$ intervals (SD1) and $\alpha-1$ or a fractal analysis of $R R$ interval complexity. A fasting blood sample was obtained to measure blood glucose and insulin and calculate the homeostasis model assessment for insulin resistance (HOMA-IR). Results: Among obese subjects HOMA-IR, LF/HF, $\alpha-1$ and SD1 values were $2.6 \pm 2.1,2.4 \pm 1.8,1.2 \pm 0.06$ and $22.5 \pm 10$ respectively. The figures for normal weight subjects were $0.5 \pm 0.1,1.3 \pm 0.2,0.9 \pm 0.3$ and $26 \pm 7.8$ respectively. Conclusions: There is an association between spectral and fractal values of heart rate variability and HOMA-IR. These results may indicate a predominance of sympathetic control of heart rate among obese subjects.

(Rev Med Chile 2015; 143: 1129-1135)

Key words: Autonomic nervous system; Heart rate; Insulin resistance; Obesity; Overweight.

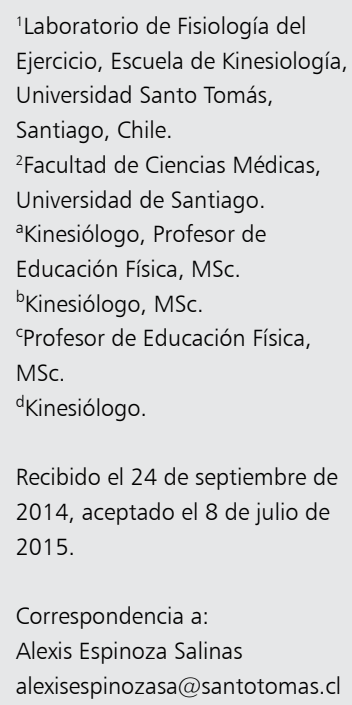

A ctualmente la población mundial enfrenta cifras importantes de obesidad, situación que además presenta una tendencia creciente en la población adulta joven ${ }^{1}$.

La obesidad ha sido asociada a alteraciones en la función del Sistema Nervioso Autónomo (SNA), demostrando la existencia de una alteración del balance autonómico sobre el nódulo sinusal ${ }^{2-4}$.

La disautonomía ha sido frecuentemente mencionada como nexo causal entre un estilo de vida poco saludable y el desbalance energético, el sedentarismo y anormalidades metabólicas como lo evidencia el síndrome metabólico ${ }^{5-7}$. En sujetos obesos se ha demostrado una disminución de la actividad vagal asociada a un mayor riesgo de enfermedad coronaria ${ }^{8}$.

Respecto a lo anterior, cobra vital importancia evaluar la función cardiaca para prevenir posibles 
patologías cardiovasculares. Un método altamente utilizado es el test de marcha de $6 \mathrm{~min}^{9}$. Sin embrago, un análisis adecuado se puede realizar mediante la medición de la Variabilidad del Ritmo Cardiaco (VRC) ${ }^{10}$, definida como la variación de latido a latido, desde un punto de vista temporal, expresando diferencias de los intervalos RR consecutivos de los complejos QRS de un electrocardiograma ${ }^{11-13}$. La VRC proporciona información cuantitativa de la modulación vagal cardiaca y de la influencia simpática ${ }^{14}$. Dentro de las metodologías utilizadas para cuantificar y analizar la VRC se encuentran los métodos temporales, espectrales y no lineales ${ }^{15}$. Estos dos últimos métodos son los utilizados en mayor medida para determinar la influencia del SNA sobre la VRC ${ }^{15,16}$. El método espectral permite analizar el contenido frecuencial de las bandas de ULF (ultra baja frecuencia), VLF (muy baja frecuencia), LF (baja frecuencia), HF (alta frecuencia) y VHF (muy alta frecuencia). Considerando los valores de LF y HF se puede establecer el cociente LF/HF para determinar el balance autonómico en reposo ${ }^{13}$.

El método no lineal cuantifica la complejidad de los intervalos RR en series de tiempo. Para este estudio se utilizó el Scattograma, que es una representación en dos dimensiones, en la cual cada intervalo RR es graficado en función del intervalo RR que lo precede, representada en este estudio a través del índice SD1 (desviación estándar de la variación instantánea de los intervalos RR $)^{15}$. El índice $\alpha-1$ representa otra medida no lineal de la VRC, un tipo de análisis fractal de la señal que mide la complejidad de los intervalos $\mathrm{RR}^{15,16}$. El análisis de estos parámetros constituye un método no invasivo para el diagnóstico de diferentes enfermedades cardiovasculares y disautonomias ${ }^{17}$.

Por otra parte, la obesidad se asocia con la resistencia a la insulina (RI). Ambas condiciones contribuyen al riesgo de complicaciones cardiovasculares ${ }^{18}$. Estableciendo un desbalance del SNA, con un predominio del sistema nervioso simpático (SNS) el cual regula las funciones metabólicas y cardiovasculares, participando en el control del metabolismo de la glucosa y las grasas ${ }^{19}$.

Estudios epidemiológicos han demostrado que la obesidad y la RI se asocian con un aumento de la frecuencia cardiaca (FC) en reposo, lo que refleja un aumento de la actividad del SNS. Estas observaciones sugieren un vínculo entre la actividad del SNS y el aumento de la RI ${ }^{20}$.
Otros trabajos, demuestran un aumento en la insulinemia de sujetos con RI, alterando gravemente el control de la actividad simpática y retirada del tono vagal del corazón ${ }^{2,3}$, produciéndose en sujetos con obesidad una reducción de la sensibilidad del nódulo sinusal a las influencias del SNA ${ }^{21}$.

El propósito de este estudio fue analizar la relación espectral y no lineal de la VRC con el índice de resistencia a la insulina HOMA (homeostasis model assessment) de pacientes obesos y con sobrepeso.

\section{Materiales y Método}

Este estudio presenta un diseño de tipo no experimental, transversal correlacional-causal.

\section{Participantes}

El estudio constó de 25 sujetos varones con sobrepeso u obesos (Grupo O), adultos, sedentarios de 20 a 30 años (media 25,4 $\pm 3,18$ años), procedentes del Centro de Ejercicio Adaptado de la Asociación Cristiana de Jóvenes (YMCA), sin otros antecedentes mórbidos diagnosticados ni reportados. Diez individuos se clasificaron como obesos y 15 como sobrepeso (IMC $=28,4 \pm 2,6 \mathrm{~kg} /$ $\mathrm{m}^{2}$ ). El grupo control (Grupo C) estaba formado por 14 individuos adultos, sanos, normopeso ( $\mathrm{IMC}=22,5 \pm 1,3)$, sedentarios de 20 a 30 años (media 21,3 $\pm 2,2$ ), Tabla 1 . El muestreo realizado fue de tipo no probabilístico por conveniencia. Los participantes menores de 20 años y mayores de 30 años, que realizaban ejercicio más de 3 veces a la semana, con hábito tabáquico activo, historial de hipertensión, o enfermedades cardiopulmonares, y que presentaban uso de fármacos con efecto en la función autonómica fueron excluidos.

Previo a la evaluación los sujetos en estudio debieron cumplir un período de $12 \mathrm{~h}$ en ayuno y haber firmado el consentimiento informado.

\section{Procedimientos}

El estudio se realizó en el laboratorio de fisiología del ejercicio de la Universidad Santo Tomás, sede Santiago. Para su implementación se utilizó un cardiotacómetro (POLAR RS800CX, Finlandia), que registra la variación de los intervalos $R R$ en el tiempo, además del software PROTRAINER 5 , el cual extrae los datos del monitor. Para cuantificar el nivel de insulina basal y glicemia, se utilizó 
Tabla 1. Descripción de la muestra estudiada

\begin{tabular}{|lccc|}
\hline & Normopeso (14 n) & Obesos (10 n) & Sobrepeso (15 n) \\
Edad (años) & $21,3 \pm 2,2$ & $27,3 \pm 2,2$ & $23,7 \pm 2,9$ \\
\hline Índice de masa corporal $\left(\mathrm{kg} / \mathrm{m}^{2}\right)$ & $22,5 \pm 1,3$ & $31,2 \pm 1,3$ & $26,7 \pm 1,5$ \\
\hline Circunferencia de cintura (cm) & $97 \pm 9,1$ & $119 \pm 2,2$ & $112 \pm 5,4$ \\
\hline Insulina (uUl/ml) & $2,5 \pm 0,9$ & $13,8 \pm 8,4$ & $9,4 \pm 11,4$ \\
Glicemia (mg/dl) & $85,7 \pm 4,5$ & $91,1 \pm 6,9$ & $90,6 \pm 7,1$ \\
HOMA & $0,53 \pm 0,1$ & $3,2 \pm 2,2$ & $2,5 \pm 3,4$ \\
LF/HF & $1,3 \pm 0,2$ & $2,6 \pm 0,9$ & $2,03 \pm 1,9$ \\
SD1 & $26 \pm 7,8$ & $14,6 \pm 4,9$ & $25,4 \pm 10,9$ \\
Alfa 1 & $0,9 \pm 0,3$ & $1,2 \pm 0,1$ & $1,1 \pm 0,2$ \\
\hline
\end{tabular}

el equipo analizador de química clínica SIEMENS ADVIA 2400. Para el cálculo del HOMA se utilizó la siguiente fórmula: HOMA IR, (insulina en ayunas $\mathrm{x}$ glucosa en ayunas)/405, expresada la insulina en $\mathrm{Uu} / \mathrm{ml}$ y la glucosa $\mathrm{mg} / \mathrm{dl}^{23}$.

Posteriormente, los sujetos fueron medidos y pesados por los investigadores para determinar el índice de masa corporal (IMC) y categorizar el nivel de obesidad. Para establecer el nivel de actividad física de los participantes se les aplicó el cuestionario simplificado de actividad física ${ }^{24}$. La evaluación de la VRC, se realizó en posición decúbito supino, registrándose los intervalos RR durante $15 \mathrm{~min}$. Al finalizar los últimos $5 \mathrm{~min}$ de la fase de reposo se realizó la toma de datos. Controlando que la frecuencia respiratoria se mantuviese en $12 \mathrm{rpm}$ durante la monitorización. Finalizado el registro, se efectuó una punción venosa a nivel antebraquial, para obtener una muestra sanguínea de $5 \mathrm{ml}$ para el posterior análisis de la glicemia e insulina. El protocolo fue evaluado y autorizado por el comité de ética de la Universidad Santo Tomás.

\section{Análisis estadístico}

Los datos obtenidos se editaron mediante el software POLAR PROTRAINER $5 \mathrm{y}$ fueron convertidos a intervalos en milisegundos y transformados a formato TXT. Dichos datos fueron analizados por el software Kubios HRV ${ }^{\circledR}$ Analysis V2.0 (Universidad de Kuopio, Finlandia) con las respectivas autorizaciones, determinado los valores del cociente LF/HF, $\alpha-1$ y SD1.

Se llevó a cabo un análisis estadístico descriptivo e inferencial. En el primero se calculó media, desviación estándar y coeficiente de variación. Para esto se empleó el software GraphPad Prism 5 para Windows ${ }^{\circledR}$. Dentro del análisis inferencial se realizó el test de normalidad Shapiro-Wilk para establecer la distribución de los datos. Se utilizó el coeficiente de correlación de Pearson para establecer el grado de asociación entre el índice HOMA y VRC. Además se realizó un análisis de regresión lineal para modelar la relación entre las dos variables en estudio.

\section{Resultados}

La Tabla 1, muestra los valores promedios y las desviaciones estándar del cociente LF/HF, $\alpha-1$ y SD1. Los resultados muestran una relación positiva entre la potencia espectral del cociente LF/ HF y el índice HOMA, presentando valores estadísticamente significativos $(r=0,56 ; p=0,0082)$ (Figura 1). Esta tendencia se observa en el grupo $\mathrm{C}$ de manera igualmente significativa $(\mathrm{r}=0,67$; $\mathrm{p}=0,008$ ) (Figura 4). Entre el componente no lineal SD1 y el índice HOMA se muestra una tendencia inversamente proporcional en el valor no lineal $(r=-0,37 ; p=0,05)$ (Figura 2). Nuevamente los datos del grupo $\mathrm{C}$ se condicen con los datos del grupo $\mathrm{O}$, presentando una relación negativa significativa entre ambas variables $(r=-0,51$; $\mathrm{p}=0,05)$ (Figura 5). Por último, existe una tendencia positiva media entre el método no lineal $\alpha-1$ y el índice HOMA $(r=0,46 ; p=0,0036)$ (Figura 3$)$. En el caso del grupo control, no se observa ninguna dependencia entre $\alpha-1$ y el índice HOMA $(\mathrm{r}=0,13 ; \mathrm{p}=0,64)($ Figura 6$)$. 


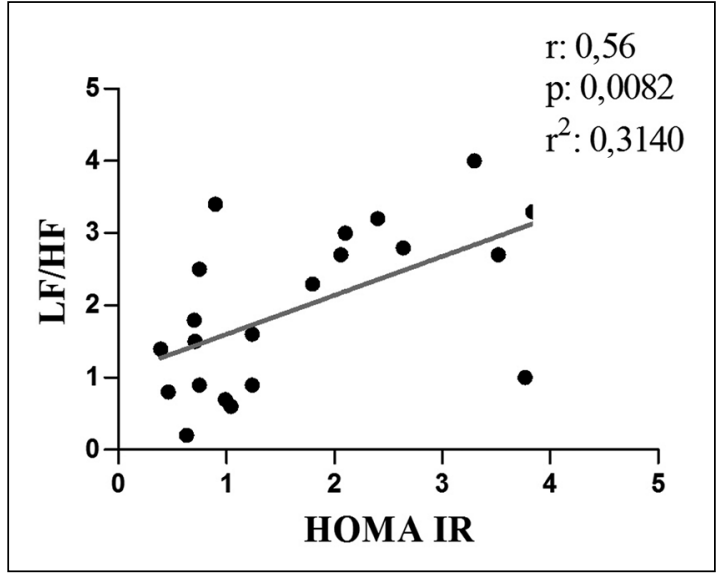

Figura 1. Potencia espectral coeficiente LF/HF y HOMA IR.

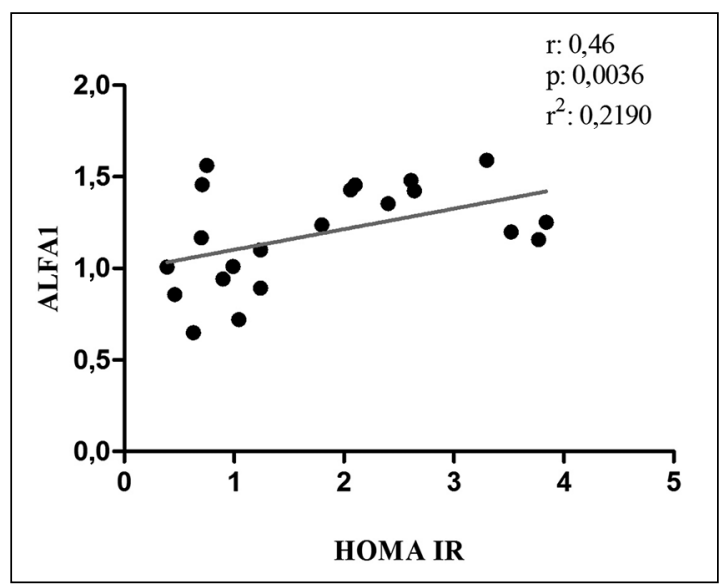

Figura 3. Método no lineal $\alpha-1$ y HOMA IR.

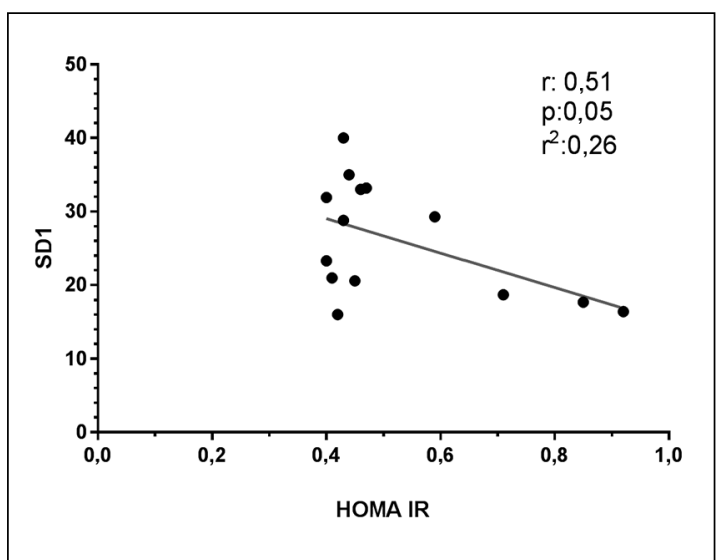

Figura 5. Componente temporal SD1 y HOMA IR de sujetos normopeso.

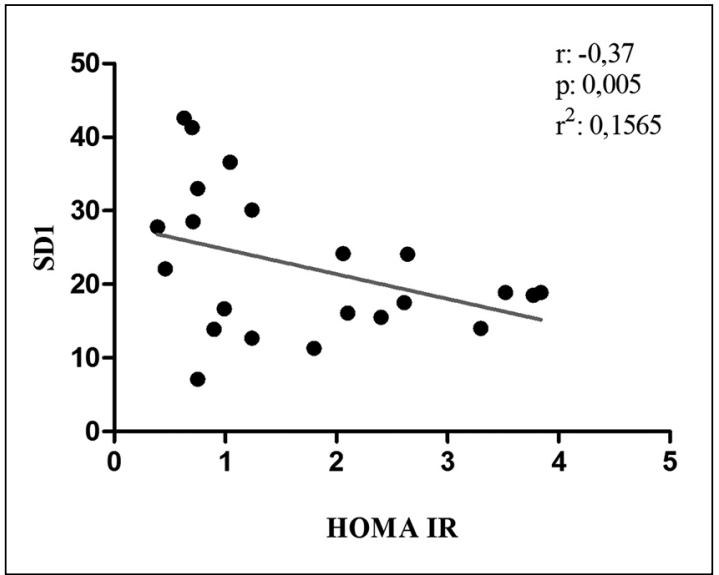

Figura 2. Componente temporal SD1 y HOMA IR.

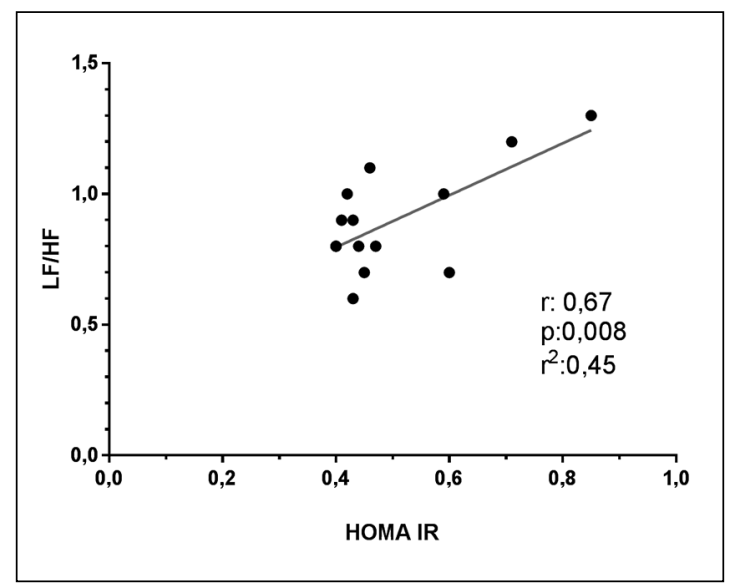

Figura 4. Potencia espectral coeficiente LF/HF y HOMA IR, sujetos normopeso.

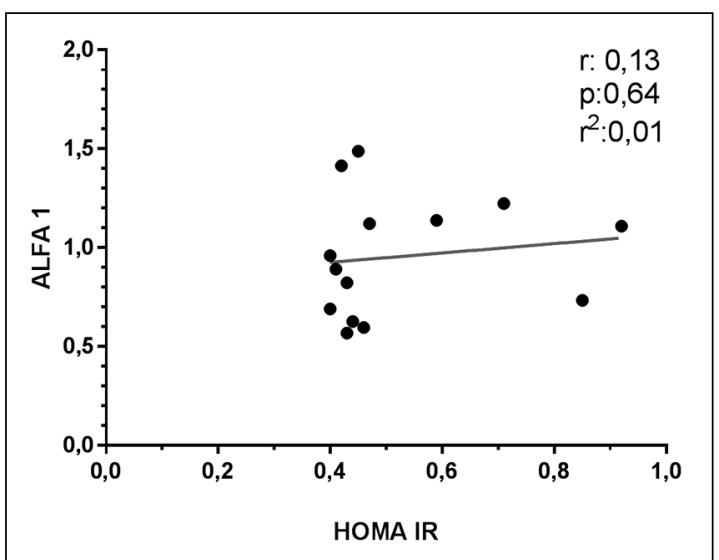

Figura 6. Método no lineal $\alpha-1$ y HOMA IR en sujetos normopeso. 


\section{Discusión}

La VRC es un método de estudio y análisis de la influencia y control autonómico sobre el comportamiento cardiovascular ${ }^{10,25,26}$. Por otra parte, la RI se define como una disminución a la respuesta fisiológica a la insulina por parte de los tejidos, particularmente a nivel del tejido muscular y adiposo ${ }^{27}$. Si se considera a la obesidad y el sobrepeso como la característica principal de la muestra del presente estudio, se presume que existe un estilo de vida poco saludable, caracterizado por el desbalance entre el gasto y el consumo calórico, constituyendo una serie de factores de riesgo para el desarrollo de anormalidades metabólicas, disautonomía y síndrome metabólico ${ }^{7,28}$.

Sumado a esto, existen estudios epidemiológicos que han demostrado que la obesidad y RI, se asocian con un incremento de la FC de reposo debido a un estímulo adrenérgico ${ }^{20}$. Esto concuerda con los resultados presentados en este estudio, que muestra una tendencia estadísticamente significativa, entre la potencia espectral determinada mediante el cociente LF/HF y el índice HOMA $(\mathrm{r}=0,56 ; \mathrm{p}=0,0082)$ (Figura 1).

Los hallazgos descritos guardan relación con los reportados por Flanagan y cols ${ }^{20}$, quien estudió en 137 sujetos la relación entre los parámetros de RI y control autonómico, encontrando una retirada de la activación vagal en hombres con RI. Dicho evento se explicaría porque en sujetos obesos, el tejido adiposo libera elevadas cantidades de adipoquinas, específicamente Factor de Necrosis Tumoral Alfa (TNF- $\alpha$ ), interleucina 6 (IL-6) y resistina, que hacen que el tejido se vuelva resistente a la acción de la insulina ${ }^{29,30}$. Una de las sustancias más importantes es el TNF- $\alpha$, el cual sería el precursor de la RI, primero porque promueve un defecto en la capacidad de fosforilación de residuos de tirosina en el primer sustrato receptor de insulina (IRS-1), necesaria para la progresión de la señal intracelular de la hormona, y segundo al disminuir la expresión génica de los transportadores de glucosa sensibles a la insulina GLUT-4.

Además, por la acción del TNF- $\alpha$, de la IL-6, de la expansión de tejido adiposo y a la aparición de RI en este el tejido, se estimula la lipasa sensible a hormonas, con lo que se beneficia la lipólisis de los triglicéridos acumulados en el tejido adiposo, acrecentando la liberación de ácidos grasos libres (AGL). Los AGL se hallan fuertemente relaciona- dos con la RI y la diabetes mellitus tipo 2. Por lo tanto, en el sujeto obeso primero aparece RI en el adipocito y luego en los otros tejidos, produciéndose un cuadro de intolerancia a la glucosa. Para tratar de regularizar la glicemia, el páncreas secreta más insulina preseverando la intolerancia a la glucosa, que puede derivar a largo plazo en diabetes mellitus tipo 2 por una disfunción de las células $\beta$ del páncreas ${ }^{27}$. Debido a esta hiperactividad para intentar mantener normales los niveles de glucosa y por el efecto lipotóxico de los AGL, se produce acumulación de cadenas largas de acetil-CoA en las células beta, y la muerte de las mismas por apoptosis.

El SNS es un importante regulador de la función cardiaca cuando hay mayor demanda metabólica de los tejidos periféricos, como ocurre durante el ejercicio. El corazón tiene abundante inervación simpática que le permite producir la mayor parte de la noradrenalina (NA) que utiliza. La NA es sintetizada en las terminaciones nerviosas y es almacenada en vesículas en el botón sináptico, protegida del poder destructor de la monoaminoxidasa que se ubica en la hendidura sináptica. Sólo una escasa cantidad de NA es producida en la médula ${ }^{31,32}$.

El aumento de la actividad simpática se puede entender por la descarga desde el nódulo sinusal, quien es el encargado de controlar la FC a través de las ramas del SNA. El aumento de la FC es una respuesta lenta al incremento de la actividad simpática, mientras que la disminución de ésta es una respuesta más temprana, que está mediada por la actividad parasimpática. Además del control central, la FC está influenciada por algunos mecanismos de feedback, como los barorreceptores arteriales, que sensan el estiramiento de las paredes vasculares por el aumento de la presión ${ }^{33}$.

Puede decirse que el SNS da una señal activadora para el miocardio que es contrabalanceada por la actividad vagal muscarínica, atenuando el efecto adrenérgico. En el SNP el neurotransmisor que actúa sobre los receptores muscarínicos es la acetilcolina. Entre ambos modulan las funciones lusitrópicas, inotrópicas y cronotrópicas del corazón. El SNS también participa en el remodelado ventricular y en la hipertrofia ventricular ${ }^{32}$.

La relación entre el desbalance autonómico y la RI aún no está clara, no obstante se ha propuesto que en sujetos obesos lo primero que se establece es un riesgo metabólico debido al exceso de grasa, 
desencadenando una RI explicado en los parrafos anteriores. El exceso de grasa generará una liberación de diferentes hormonas (adiponectina, resistina, leptina y visfatina), en conjunto con citoquinas inflamatorias (TNF- $\alpha$ y IL-6), desencadenando una activación simpática por estimulación directa del hipotálamo. Sin embargo, se cree que la vía principal por la cual esta RI genera el desbalance autonómico es la estimulación de la insulina a nivel central y periférico sobre el sistema nervioso simpático ${ }^{30}$. Esta hipótesis es respaldada por el trabajo de Wu y cols. en la cual se somete a una cirugía bariátrica a pacientes obesos, con RI y desbalance autonómico. Los resultado demuestran una disminución significativa del índice HOMA en un período de 90 días, y posteriormente un reestablecimiento del balance autonómico, respaldando la hipótesis de que primero se instala la RI, y como consecuencia la hiperinsulinemia, generando un desbalance autonómico secundario ${ }^{34}$.

Las variaciones más notorias se observaron en los valores de LF/HF, lo cual indica una retirada vagal con predomino del SNS, presentando un comportamiento similar a los estudios de $\mathrm{Wu}$ y cols. ${ }^{34}$ y Hillebrand y cols. ${ }^{30}$. Por otro lado, estos hallazgos se contradicen con los resultados encontrados en el grupo $\mathrm{C}$, donde esta relación positiva se establece de igual manera, poniendo en tela de juicio que el motivo de esta retirada vagal se deba exclusivamente a los mecanismos fisiológicos expuestos anteriormente.

El comportamiento de $\alpha-1$ no evidencia los resultados esperados para el grupo $\mathrm{O}^{10,35}$, presentando una tendencia hacia el incremento de la complejidad de la señal respecto a los valores del HOMA IR (Figura 3). No obstante, los hallazgos descritos guardan relación con los reportados por Huikuri y cols ${ }^{36}$. Esto podría explicarse por la existencia de otros factores que predominan sobre el estatus clínico en estudio, que modificarían los efectos de las características individuales previas. Por lo tanto, se podría esperar algún tipo de efecto cardioprotector, como por ejemplo la práctica de actividad física regular suspendida meses antes del estudio y que no fue reportada por los participantes. Además este ítem no está incluido en el cuestionario simplificado de actividad física.

Hasta el momento no existen estudios que hayan establecido todas las variables que pueden modificar los valores de $\alpha-1$, por lo que es necesario generar nuevas líneas de investigación en esta área que nos permitan explicar tal comportamiento. No obstante, los valores del análisis espectral confirman un predominio simpático sobre la función cardiovascular.

\section{Referencias}

1. Han E, Powell LM. Effect of food prices on the prevalence of obesity among young adults. Public health 2011; 125 (3): 129-35.

2. Windham B, Fumagalli S, Ble A, Sollers J, Thayer J, Najjar S, et al. The Relationship between Heart Rate Variability and Adiposity Differs for Central and Overall Adiposity. Journal of Obesity 2012; 2012: 1-8.

3. Straznicky N, Grima M, Sari C, Eikelis N, Lambert G, Nestel P, et al. A Randomized Controlled Trial of the Effects of Pioglitazone Treatment on Sympathetic Nervous System Activity and Cardiovascular Function in Obese Subjects With Metabolic Syndrome. The Journal of Clinical Endocrinology \& Metabolism 2014; 99 (9): E1701-7.

4. Muralikrishnan K, Balasubramanian K, Ali SM. Poincare. Plot of heart rate variability: an approach towards explaining the cardiovascular autonomic function in obesity. Indian J Physiol Pharmacol 2013; 57 (1): 31-7.

5. Lips MA, de Groot GH, De Kam M, Berends FJ, Wiezer $\mathrm{R}$, Van Wagensveld BA, et al. Autonomic nervous system activity in diabetic and healthy obese female subjects and the effect of distinct weight loss strategies. Eur J Endocrinol 2013; 169 (4): 383-90.

6. Molfino A, Fiorentini A, Tubani L, Martuscelli M, Rossi Fanelli F, Laviano A. Body mass index is related to autonomic nervous system activity as measured by heart rate variability. Eur J Clin Nutr 2009; 63 (10): 1263-5.

7. Licht C, de Geus E, Penninx B. Dysregulation of the Autonomic Nervous System Predicts the Development of the Metabolic Syndrome. The Journal of Clinical Endocrinology \& Metabolism 2013; 98 (6): 2484-93.

8. Broom I, Wilding J, Stott P, Myers N. Randomised trial of the effect of orlistat on body weight and cardiovascular disease risk profile in obese patients: UK Multimorbidity Study. Int j Clin pract 2002; 56 (7): 494-9.

9. AACVPR Guidelines for cardiac rehabilitation and secondary prevention programs. 4th ed. Champaing: Human Kinetics; 2004.

10. Task Force. Heart Rate Variability: Standards of Measurement, Physiological Interpretation, and Clinical Use. Circulation 1996; 93: 1043-65.

11. Karason K, Molgaard H, Wikstrand J, Sjöström L. Heart rate variability in obesity and the effect of weight loss. Am J Cardiol 1999; 83 (8): 1242-51. 
12. Goldberger J. Sympathovagal balance: how should we measure it? Am J Physiol 1999; (45): H1273-80.

13. Mietus JE, Peng C, Henry I, Goldsmith R, Goldberger A. Thep NNx files: re-examining a widely used heart rate variability measure. Heart 2002; 88: 378-80.

14. Gang Y, Malik M. Heart rate variability Analysis in General Medicine. Indian Pacing Electrophysiol J 2003; 1: 34-40.

15. Jeria C, Hernández R, Benn C. Alteración de la variabilidad del ritmo cardiaco en pacientes con síndrome coronario agudo sin supradesnivel del segmento ST: Experiencia preliminar. Rev Chil Cardiol 2011; 31: 10412.

16. Gomis P, Canimal P, Vallverdu M, Wagner G. Análisis Fractal del sistema de control cardiaco durante la isquemia de miocardio. En: Conferencia XXV jornada de automática. Cataluña, España. 2004.

17. Ruiz Y, Lorenzo J, Taboada A. Extracción y análisis de la señal de la variabilidad de ritmo cardiaco (HRV) mediante filtrado. I.E.E.A.C. 2005; 26: 31-6.

18. Emdin M, Gastaldelli A, Muscelli E, Macerata A, Natali A, Camastra S. Hyperinsulinemia and Autonomic Nervous System Dysfunction in Obesity: Effects of Weight Loss. Circulation 2001; 103: 513-9.

19. Berne R, Levy M. Fisiología. Ed Elsevier. Madrid. España. 2006; (4): 344-59.

20. Flanagan D, Vaile J, Petley G, Moore V, Godsland I, Cockington R. The Autonomic Control of Heart Rate and Insulin Resistance in Young Adults. J Clin Endocrinol Metab 1999; (84): 1263-7.

21. Kuller L, Eichner J, Orchard T, Grandits G, McCallum L, Tracy R. The relation between serum albumin levels and risk of coronary heart disease in the Multiple Risk Factor Intervention Trial. Am J Epidemiol 1991; 134: 1266-77.

22. Hernández R, Fernández C, Baptista P. Metodología de la Investigación. Ed. Mc Graw Hill. México. 2006; 3: 234-56.

23. Acosta A, Escalona M, Maiz A, Pollak F, Leighton F. Determinación del índice de resistencia insulínica mediante HOMA en una población de la Región Metropolitana de Chile. Rev Med Chile 2002; 130: 1227-31.

24. Ainsworth B, Jacobs D, Leon A. Validity and reliability of self-reported physical activity status: the Lipid Research Clinics questionnaire. Medicine \& Science in
Sports \& Exercise 1993; 25 (1): 92-8.

25. Sztajzel J. Heart rate variability: a noninvasive electrocardiographic method to measure the autonomic nervous system. Swiss Med Wkly 2004; 134: 514-22.

26. Lombardi F. Chaos theory, heart rate variability, and arrhythmic mortality. Circulation 2000; 101: 8-10.

27. Rodríguez-Rodríguez E, Perea J, López-Sobaler A, Ortega R. Obesidad, resistencia a la insulina y aumento de los niveles de adipoquinas: importancia de la dieta y el ejercicio físico. Nutr Hosp 2009; 24 (4): 415-21.

28. Bobbioni-Harsch E, Sztajzel J, Barthassat V, Makoundou V, Gastaldi G, Sievert K, et al. Independent evolution of heart autonomic function and insulin sensitivity during weight loss. Obesity (Silver Spring) 2009; 17 (2): 247-53.

29. González CA, Malanco HLM, Sánchez ZMJ, Elizondo AS, Navarro ZJE, Rosillo RS. Inflamación y resistencia a la insulina: Mecanismos para el desarrollo de la disfunción endotelial y aterosclerosis. Rev Mex Cardiol 2006; 17 (2): 71-82.

30. Hillebrand S, Swenne CA, Gast KB, Maan AC, le Cessie $\mathrm{S}$, Jukema JW, et al. The role of insulin resistance in the association between body fat and autonomic function. Nutr Metab Cardiovasc Dis. Elsevier B.V 2015; 25 (1): 93-9.

31. Navarro X. Fisiología del sistema nervioso autónomo. Rev Neurol 2002; 6 (35): 553-62.

32. López J, Fernández A. Fisiología del ejercicio. Buenos Aires, Madrid: Médica Panamericana 2006; 3: 326-71.

33. Tarvainen M, Niskanen J. Kubios HRV Versión 2.0 User's Guide. Biosignal Analisys and Medical Imaging Group Department of Physics University of Kuopio. 2008; $2: 9$.

34. Wu J, Yu H, Lai H, Yang P, Lin M, Lai F. Improvement of heart rate variability after decreased insulin resistance after sleeve gastrectomy for morbidly obesity patients. Surgery for Obesity and Related Diseases. 2014.

35. Kleiger R, Stein P, Bigger T. Heart rate variability: measurement and clinical utility. A. N. E. 2006; 10: 88-10.1.

36. Huikuri HV, Makikallio TH, Peng CK, Goldberger AL, Hintze U, Moller M. Fractal correlation properties of $\mathrm{R}-\mathrm{R}$ interval dynamics and mortality in patients with depressed left ventricular function after an acute myocardial infarction. Circulation 2000; 101: 47-53. 